\title{
Measurement of Use of Electronic Resources: Advances in Use Statistics and Innovations in Resource Functionality
}

\author{
Deborah D. Blecic, Joan B. Fiscella, and Stephen E. \\ Wiberley, Jr.
}

The ICOLC guidelines and Project COUNTER codes of practice have advanced the measurement of use of electronic resources. At the same time, innovations in functionality within and among electronic resources are changing the environment in which use is measured. The present article explores measures of sessions and searches for one research library's electronic resources. The article analyzes the transition from vendor-specific to COUNTER-compliant statistics, how vendors measure the running of search alerts, and the effects of federated searching on reported use. The analysis suggests that innovations in functionality may have changed the meaning of sessions and searches. The analysis also suggests the following principle: innovations in electronic resource functionality will necessitate advances in electronic resource usage measures to describe use meaningfully.

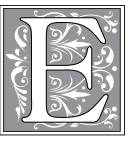

lectronic resources have become an increasingly substantial component of academic library collections over the last decade. Mary M. Case has reported that "Between 1994/95 and 2001/02, expenditures on electronic resources for the typical university research library have grown almost $400 \% .{ }^{\prime 1}$ In 2004, the median expenditure for electronic materials in ARL libraries was over $\$ 2,600,000 .^{2}$ Often, the expenditures for a resource only ensure access for a limited time period rather than ownership/perpetual access. Annual licenses may carry fees of five or even six figures. Given the high stakes, it is vital for libraries to have meaningful measurements of the use of electronic resources to inform prudent expenditure of limited collections budgets. In recent years, methods used to measure use of electronic resources have evolved and are continuing to do so via initiatives such as the International Coalition of Library Consortia (ICOLC) guidelines and Project COUNTER (Counting Online Usage

Deborah D. Blecic is Bibliographer for the Life and Health Sciences and Associate Professor, Joan B. Fiscella is Interim Principal Bibliographer and Associate Professor, and Stephen E. Wiberley, Jr. is Bibliographer for the Social Sciences and Professor at the University of Illinois at Chicago; e-mail: dblecic@uic.edu, jbf@uic. edu, and wiberley@uic.edu, respectively. The authors wish to thank Mary Case, John Cullars, and Nancy John for their helpful reviews of this manuscript. 
of NeTworked Electronic Resources) codes of practice. In 2004, the Association of Research Libraries began to collect use data of electronic resources for its supplementary statistics. ${ }^{3}$ At the same time that publishers and librarians were improving the recording and gathering of use statistics, developments in electronic resources functionality such as federated searching and the increased availability of alerting services were enabling patrons to use electronic resources in new ways. As James Pringle has observed, "even as the reasons for gathering and disseminating usage statistics have multiplied, the phenomenon measured (i.e., user behavior in electronic environments) has become more complex." 4

\section{Study Overview}

The present study grew out of a multiyear effort to analyze the use statistics available for electronic resources licensed by the study library. The study library serves a research university that offers 87 bachelor's, 88 master's, and 58 doctoral programs, with nearly 25,000 students and over 1,600 faculty. Between FY2000 and FY2004 the library's electronic resources budget grew from $\$ 475,000$ to $\$ 2,045,260$. The authors examined data from three fiscal years (July 1-June 30), 2002 to 2004. The authors noted differences in reported data with the adoption of COUNTER-compliant statistics and other differences with the use of search alert services and federated searching. The present study examines the possible effects of 1) COUNTER-compliant statistics, 2) search alert services, and 3) federated searching on vendor-supplied search and session data. Each section of the study highlights different electronic resources that illustrate a particular advance or innovation.

Understanding the possible meanings of the terms "sessions" and "searches" is essential when interpreting use statistics. The ICOLC guidelines recognized the terms as important measures of use. In 1998 and 2001, the guidelines did not de- fine "session," but equated it to "logins." 5,6 In both its first and second releases, The COUNTER Code of Practice for Journals and Databases defined a session as "a successful request of an online service. It is one cycle of user activities that typically starts when a user connects to the service or database and ends by a terminating activity that is either explicit (by leaving the service through exit or logout) or implicit (timeout due to user inactivity)." 7,8 In other words, a connection to an online service constitutes a session. Whether a user has to initiate any activity in addition to connecting to an online service to incur a session count is an important question. Appendix E of Release 2 of the COUNTER Code of Practice for Journals and Databases provides that "a database will only get credit for the session if it has been searched during that session." 9 The authors could not determine if that assignment of credit refers to all scenarios or only those in which a platform has multiple databases that may be searched separately. The authors have observed numerous instances of use statistics where numbers of sessions reported exceed numbers of searches reported. This indicates it is possible to have a session-i.e., a connection to a database or other service-during which there is no search. Among the possible scenarios for a "searchless session" are these: 1) users connect to databases that they navigate via mouse clicks rather than searches; 2 ) users connect to an article database by clicking on a link that opens an article in that database; 3 ) users connect to a service but terminate activity before searching because they realize they have accessed the wrong service; 4) users connect to a resource, then click on information about the service and terminate the session before searching.

ICOLC defined a search (query) as "a unique intellectual inquiry. Typically a search is recorded each time a search form is sent/submitted to the server. "10 Project COUNTER has used a very similar definition of a search: "a specific intellectual 
query, typically equated to submitting the search form of the online service to the server." ${ }^{11}$ The authors recognize that, before a vendor becomes COUNTERcompliant, what constitutes a session or a search reported by that vendor for its online services depends on the operational definition of a session or a search used by that vendor's statistical software.

\section{COUNTER-compliant Statistics}

In the early stages of electronic resource use statistics, each vendor reported statistics in its own way. One early attempt to standardize measures was the ICOLC initiative to identify and define key elements of use. ICOLC recommended that five elements - sessions, items, queries (searches), menu selections, and turnaways - be included in any vendor report. ${ }^{12}$ Experience showed, however, that different vendors and different data collection software could have different definitions of what constituted these elements (e.g., the meaning of a session for one vendor could differ from the meaning used by another) $\cdot{ }^{13}$ Each vendor may have also defined a search in a slightly different way or analyzed searching into various subcategories. ${ }^{14,15}$ Thus, measures that were nominally the same in actuality measured use differently. In response to this uncertainty, Project COUNTER emerged as an organized effort to standardize how the elements are counted.

COUNTER-compliant statistics use some of the same data elements defined by ICOLC, but go a step further in attempting to ensure that all vendors count the data elements consistently. ${ }^{16}$ "COUNTER has been developed to provide a single, international, extendible Code of Practice that allows the usage of online information products and services to be measured in a credible, consistent, and compatible way using vendor-generated data." ${ }^{17}$ This uniformity enables librarians to have greater confidence that statistics from different vendors represent a similar type of use. Requiring publishers to submit usage reports for auditing before claiming COUNTER compliance adds to the credibility. ${ }^{18}$

The COUNTER project continues to evolve. Release 2 of the Code of Practice for Journals and Databases went into effect on January 1, 2006. Release 2 does not expand the types of statistics detailed in Release 1, but rather "the majority of the changes in Release 2 are designed to provide more specific information to assist vendors to create COUNTER-compliant usage reports, and to enable customers to use them." ${ }^{19}$ The consistency and ease of use of COUNTER-compliant reports will facilitate data gathering by libraries.

As COUNTER develops, vendors are switching to COUNTER-compliant usage reports at varying times. As of July 2004, thirteen of forty-two vendors examined for this study had released at least one COUNTER-compliant statistics report for at least one resource. For clarity in discussion, the authors will call preCOUNTER or non-COUNTER usage statistics "vendor-specific statistics." The authors found that for some resources, use data were available for the same time period as both vendor-specific and COUNTER-compliant reports. Some vendors reported both COUNTER-compliant and vendor-specific statistics for the time period in which they made the transition to COUNTER-compliant reports. Other vendors were able to run COUNTERcompliant statistics software against transaction logs that dated back months before project COUNTER was organized. The availability of COUNTER-compliant data that overlapped vendor-specific data enabled the authors to compare the differences between COUNTER-compliant and vendor-specific statistics for various vendors.

The differences between vendor-specific and COUNTER-compliant statistics vary among vendors. The differences range from completely unrelated - that is, vendor-specific statistics seem to have no similarity to COUNTER-compliant statistics - to no difference at all. Table 1 illustrates the range of differences for searches 
Advances in Use Statistics and Innovations in Resource Functionality 29

\begin{tabular}{|c|c|c|c|c|c|c|c|c|c|c|c|c|}
\hline & \multirow{2}{*}{ 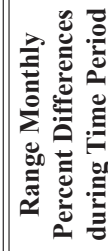 } & 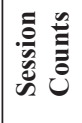 & $\begin{array}{l}\stackrel{8}{0} \\
\stackrel{0}{0} \\
=\end{array}$ & $\begin{array}{ll}0 & 8 \\
0 & 2 \\
1 & =\end{array}$ & 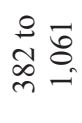 & 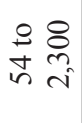 & \begin{tabular}{l}
$\stackrel{0}{8} 8$ \\
$\stackrel{2}{\circ}$ \\
\hdashline \\
1
\end{tabular} & $\begin{array}{l}0 \\
\stackrel{8}{8} \\
0 \\
0\end{array}$ & $\frac{0}{i} \frac{n}{n}$ & 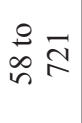 & $\begin{array}{cc}0 & 8 \\
i & 0 \\
1 & 0 \\
1\end{array}$ & $\stackrel{\circ}{8} \underset{1}{\circ}$ \\
\hline & & 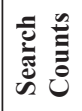 & 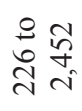 & $\begin{array}{l}\stackrel{0}{\circ} \\
2 \\
2\end{array}$ & 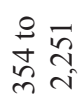 & 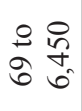 & 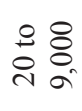 & $\begin{array}{l}\stackrel{0}{2} \\
\stackrel{2}{2} \\
\stackrel{2}{2}\end{array}$ & 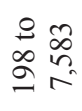 & $\begin{array}{l}\stackrel{2}{a} \\
\sim \\
\tilde{m} \\
m\end{array}$ & 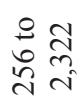 & $\begin{array}{l}\stackrel{\ominus}{*} \\
\stackrel{\infty}{\Xi} \\
=\end{array}$ \\
\hline \multirow{2}{*}{ 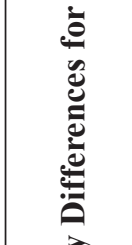 } & \multirow{2}{*}{ 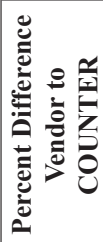 } & 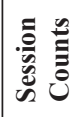 & $\stackrel{m}{\forall}$ & \&े & $\underset{\infty}{+\infty}$ & $\ddot{\infty}$ & $\sqrt[\widetilde{n}]{\sim}$ & in & 宇 & $\underset{m}{m}$ & $\stackrel{n}{\frac{n}{f}}$ & ஜ̊ \\
\hline & & Ũ & $\stackrel{n}{f}$ & $\underset{\sim}{\stackrel{\sim}{\sim}}$ & $\stackrel{\infty}{\sim}$ & $\stackrel{\curvearrowright}{\Delta}$ & ஓे & ర্ঠ & $\underset{\infty}{\infty}$ & $\underset{n}{J}$ & $\underset{m}{\stackrel{J}{*}}$ & ì \\
\hline \multirow{5}{*}{ 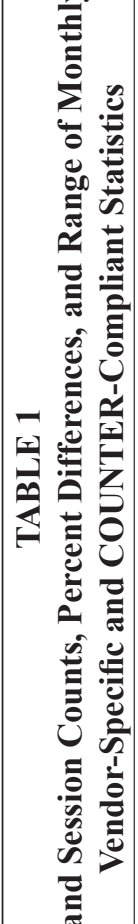 } & 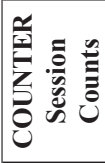 & & $\begin{array}{l}0 \\
\stackrel{2}{2} \\
\end{array}$ & $\begin{array}{l}\overline{6} \\
+ \\
i\end{array}$ & $\begin{array}{l}\hat{\sigma} \\
\text { જे }\end{array}$ & $\infty$ & के & 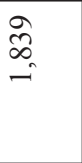 & $\begin{array}{l}\hat{n} \\
\text { nn } \\
i\end{array}$ & 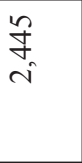 & $\stackrel{n}{0}$ & $\vec{f}$ \\
\hline & 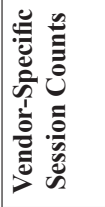 & & ત̃ & $\overline{8}$ & ֻે & $\stackrel{\infty}{+}$ & $\infty$ & $\bar{q}$ & $\hat{\tilde{n}}$ & $\frac{n}{n}$ & $\infty$ & $\stackrel{\Xi}{\sim}$ \\
\hline & 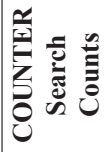 & & $\begin{array}{l}\stackrel{0}{\circ} \\
\text { ஸे } \\
\infty \\
\infty\end{array}$ & $\begin{array}{l}8 \\
\infty \\
\infty \\
\text { ri }\end{array}$ & $\begin{array}{l}2 \\
i n \\
n\end{array}$ & $\hat{尺}$ & ڤ్రి & $\begin{array}{l}\sqrt{n} \\
\tilde{\sigma} \\
\text { i }\end{array}$ & $\begin{array}{l}\text { ते } \\
\text { ñ }\end{array}$ & $\begin{array}{l}\stackrel{\infty}{\infty} \\
\stackrel{+}{+}\end{array}$ & $\begin{array}{l}\infty \\
\stackrel{0}{0} \\
i\end{array}$ & $\begin{array}{l}\infty \\
\stackrel{\infty}{0} \\
\end{array}$ \\
\hline & 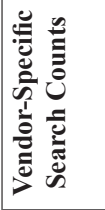 & & ڤે & $\stackrel{\infty}{\sim}$ & $\begin{array}{l}\stackrel{m}{f} \\
\stackrel{v}{v}\end{array}$ & $\stackrel{n}{\text { ¿ }}$ & \&̊ & iे & $\stackrel{\infty}{0}$ & $\hat{\mathfrak{b}}$ & $\stackrel{\sim}{\sim}$ & $\stackrel{m}{\vec{\gamma}}$ \\
\hline & 总 & & 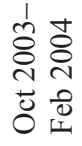 & 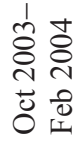 & 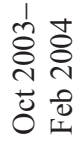 & 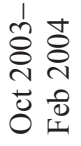 & 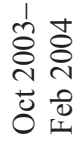 & 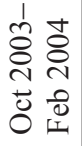 & 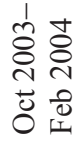 & 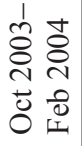 & 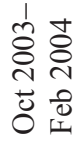 & 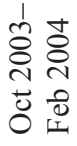 \\
\hline 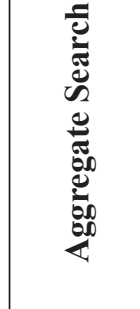 & 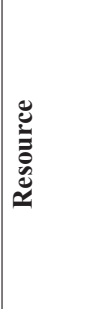 & & 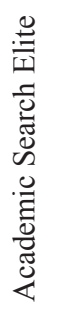 & 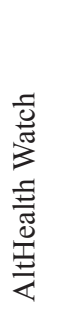 & 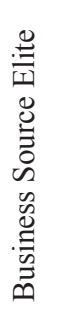 & 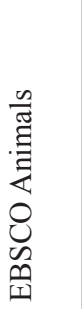 & $\frac{U}{\frac{U}{I}}$ & 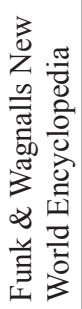 & 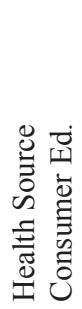 & 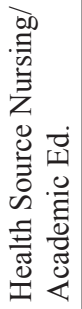 & 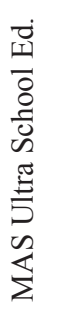 & 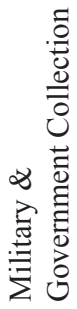 \\
\hline & $\begin{array}{l}\dot{\bar{\theta}} \\
\stackrel{0}{0} \\
\dot{0}\end{array}$ & & $\begin{array}{l}0 \\
\text { Un } \\
0 \\
0 \\
\text { II }\end{array}$ & $\begin{array}{l}0 \\
0 \\
\mathscr{W} \\
0 \\
0\end{array}$ & 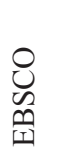 & $\begin{array}{l}0 \\
\mathscr{U} \\
\mathscr{P} \\
\text { II }\end{array}$ & $\begin{array}{l}0 \\
\text { D } \\
\text { صิ } \\
\text { II }\end{array}$ & $\begin{array}{l}0 \\
\text { ڤn } \\
\text { صิ } \\
\text { II }\end{array}$ & 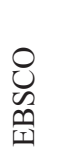 & $\begin{array}{l}0 \\
\text { ڤn } \\
\text { صิ } \\
\text { II }\end{array}$ & $\begin{array}{l}0 \\
0 \\
\mathscr{D} \\
\stackrel{1}{\mid c}\end{array}$ & $\begin{array}{l}0 \\
\text { D } \\
\text { صิ } \\
\text { II }\end{array}$ \\
\hline
\end{tabular}




\begin{tabular}{|c|c|c|c|c|c|c|c|c|c|c|c|}
\hline & \multirow{2}{*}{ 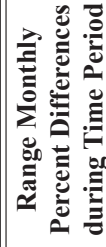 } & 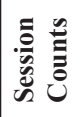 & 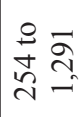 & $\stackrel{0}{8}$ & 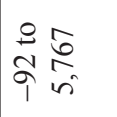 & $\stackrel{0}{8} 8$ & $\overleftrightarrow{Z}$ & 0 & 0 & 0 & 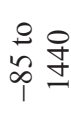 \\
\hline & & 吾 & $\begin{array}{ll}0 & \infty \\
2 & 0 \\
\infty & -\end{array}$ & \begin{tabular}{ll}
8 & 0 \\
\multirow{2}{*}{} & $\stackrel{?}{\sim}$ \\
$\sim$ & $=$
\end{tabular} & 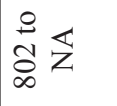 & 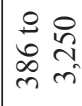 & $\begin{array}{l}\infty \\
\stackrel{2}{-}\end{array}$ & 0 & 0 & 0 & $\stackrel{0}{2} \hat{\infty}$ \\
\hline \multirow{2}{*}{ 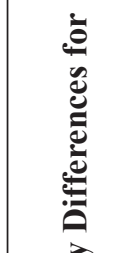 } & \multirow{2}{*}{ 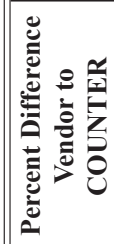 } & 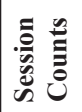 & $\stackrel{\wp}{f}$ & $\stackrel{\text { qे }}{m}$ & $\stackrel{i}{n}$ & $\bar{n}$ & $\overleftrightarrow{Z}$ & 0 & 0 & 0 & $\stackrel{\sim}{\sim}$ \\
\hline & & 竞 & $\underset{\sim}{\stackrel{\sim}{\sim}}$ & $\underset{\sim}{0}$ & $\frac{a}{n}$ & $\hat{\circ}$ & $\sim$ & 0 & 0 & 0 & $\stackrel{\vartheta}{\imath}$ \\
\hline \multirow{7}{*}{ 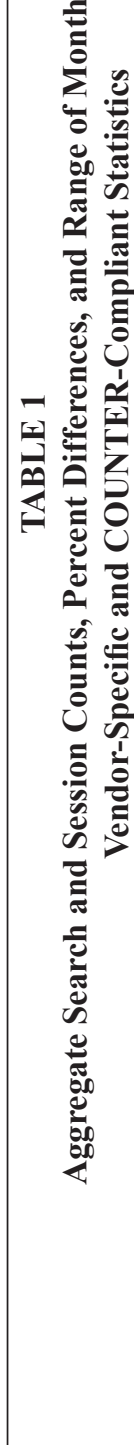 } & 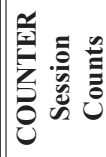 & & 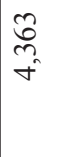 & $\begin{array}{l}\infty \\
\stackrel{\infty}{\sim} \\
-1\end{array}$ & 홍 & $\underline{6}$ & $\overleftrightarrow{Z}$ & $\begin{array}{l}\infty \\
\infty \\
\infty \\
0\end{array}$ & $\stackrel{\Xi}{\vec{F}}$ & $\begin{array}{l}0 \\
\varrho \\
0 \\
0\end{array}$ & $\stackrel{n}{\infty}$ \\
\hline & 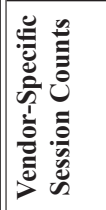 & & $\stackrel{\infty}{\infty}$ & 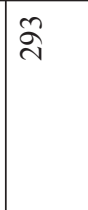 & 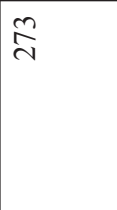 & $\hat{n}$ & $\overleftrightarrow{Z}$ & $\begin{array}{l}\infty \\
\infty \\
\infty \\
n\end{array}$ & $\stackrel{\nabla}{\vec{F}}$ & $\begin{array}{l}0 \\
\varrho \\
0\end{array}$ & $\stackrel{\infty}{\sigma}$ \\
\hline & 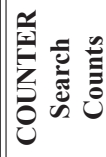 & & $\frac{\circ}{2}$ & $\begin{array}{l}\text { in } \\
\infty \\
i \\
i\end{array}$ & $\frac{8}{m}$ & ๙ે & $\frac{\infty}{\circ}$ & $\begin{array}{l}\tilde{\alpha} \\
\hat{\sigma}\end{array}$ & $\begin{array}{l}\text { gे } \\
\text { +े }\end{array}$ & $\frac{8}{2}$ & 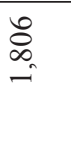 \\
\hline & 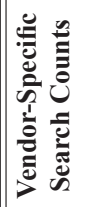 & & $\begin{array}{l}8 \\
0 \\
i\end{array}$ & $\frac{\pi}{6}$ & $\frac{n}{n}$ & $\tilde{n}$ & \begin{tabular}{l}
$\infty$ \\
$\stackrel{\infty}{*}$ \\
\multirow{8}{\delta}{}
\end{tabular} & $\begin{array}{l}\hat{\sigma} \\
\hat{\sigma}\end{array}$ & $\begin{array}{l}\stackrel{\partial}{\partial} \\
\dot{+}\end{array}$ & $\frac{8}{2}$ & \begin{tabular}{c}
\multirow{4}{*}{} \\
$\stackrel{4}{1}$
\end{tabular} \\
\hline & : & & 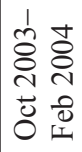 & 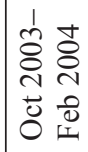 & 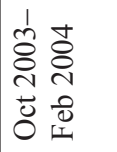 & 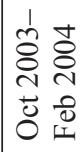 & 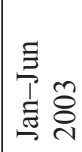 & 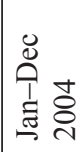 & 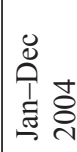 & 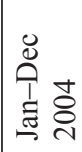 & 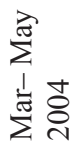 \\
\hline & 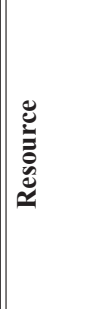 & & 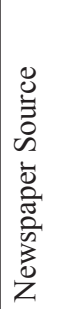 & 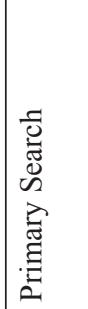 & 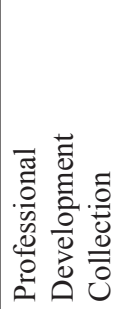 & 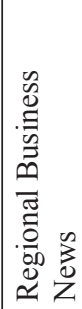 & 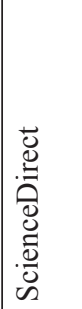 & 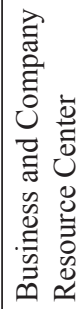 & 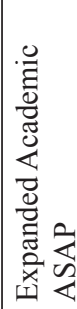 & 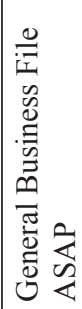 & 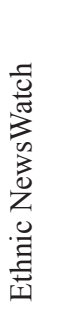 \\
\hline & 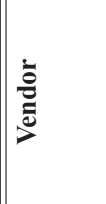 & & $\begin{array}{l}0 \\
\text { Un } \\
0 \\
\text { II }\end{array}$ & $\begin{array}{l}0 \\
\text { On } \\
0 \\
01\end{array}$ & $\begin{array}{l}0 \\
\text { W } \\
0 \\
0\end{array}$ & $\begin{array}{l}0 \\
\text { D } \\
\infty \\
0 \\
1\end{array}$ & 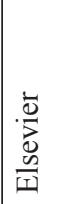 & 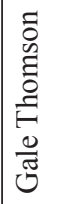 & $\begin{array}{l}\tilde{0} \\
0 \\
0 \\
0 \\
0 \\
10 \\
\frac{0}{\pi} \\
0 \\
0\end{array}$ & 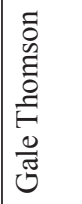 & 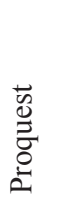 \\
\hline
\end{tabular}


Advances in Use Statistics and Innovations in Resource Functionality 31

\begin{tabular}{|c|c|c|c|c|c|c|c|}
\hline \multirow{11}{*}{ 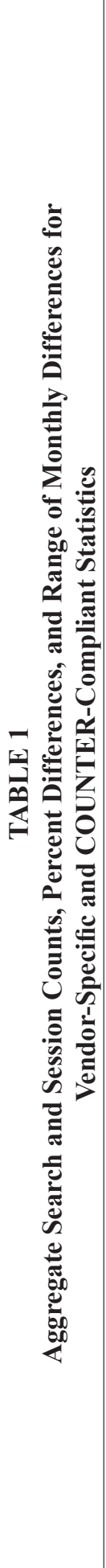 } & \multirow{2}{*}{ 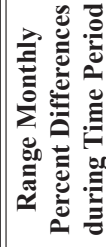 } & 昜 & 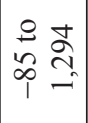 & $\begin{array}{l}= \\
0 \\
0\end{array}$ & 0 & 0 & 0 \\
\hline & & 造 & 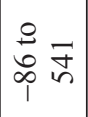 & $\begin{array}{l}1 \\
\stackrel{1}{0} \\
0\end{array}$ & 0 & 0 & 0 \\
\hline & \multirow{2}{*}{ 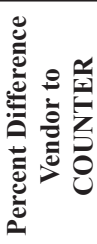 } & 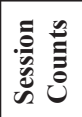 & $\stackrel{\infty}{\sim}$ & $N$ & 0 & 0 & 0 \\
\hline & & 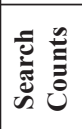 & $\stackrel{p}{i}$ & N & 0 & 0 & 0 \\
\hline & 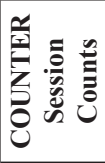 & & $\stackrel{n}{\infty}$ & $\begin{array}{l}\stackrel{P}{n} \\
\text { n. }\end{array}$ & बे & 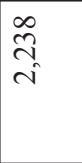 & $\underset{m}{\stackrel{0}{m}}$ \\
\hline & 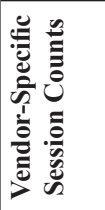 & & $\begin{array}{l}n \\
i \\
i\end{array}$ & $\begin{array}{l}8 \\
\stackrel{8}{ } \\
\text { in }\end{array}$ & Әे & $\begin{array}{l}\infty \\
\underset{\sim}{\sim} \\
\text { i }\end{array}$ & $\underset{m}{\stackrel{0}{m}}$ \\
\hline & 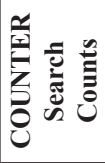 & & $\underset{\infty}{\infty}$ & $\underset{\sigma}{\sigma}$ & $\begin{array}{l}\stackrel{2}{2} \\
\text { ì }\end{array}$ & $\frac{\infty}{\sigma}$ & $\frac{i}{\stackrel{2}{r}}$ \\
\hline & 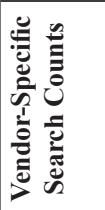 & & $\begin{array}{l}\hat{\infty} \\
0 \\
i\end{array}$ & $\begin{array}{l}\sqrt{2} \\
\text { ñ. } \\
b\end{array}$ & $\begin{array}{l}\stackrel{2}{2} \\
\text { ì }\end{array}$ & $\begin{array}{l}\infty \\
\stackrel{\sigma}{\sigma}\end{array}$ & $\frac{i}{a}$ \\
\hline & 窇 & & 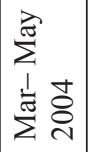 & 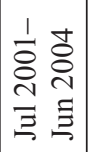 & 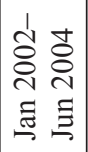 & 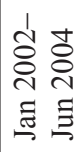 & 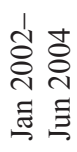 \\
\hline & 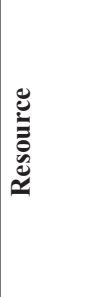 & & $\begin{array}{l}\frac{1}{0} \\
\frac{\pi}{\pi} \\
\frac{0}{0} \\
\frac{0}{0} \\
0\end{array}$ & 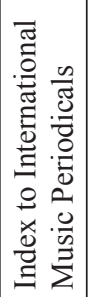 & 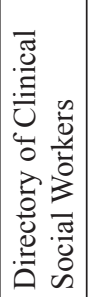 & 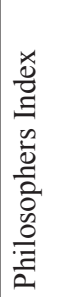 & 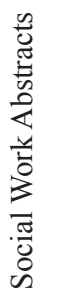 \\
\hline & $\begin{array}{l}\dot{\bar{\theta}} \\
\frac{0}{0}\end{array}$ & & 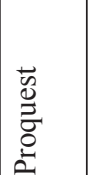 & 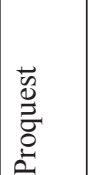 & 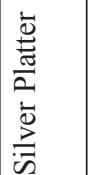 & 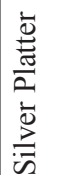 & 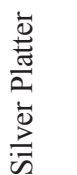 \\
\hline
\end{tabular}

and sessions during time periods when vendors reported both vendorspecific and COUNTERcompliant searches and sessions, showing the aggregate totals and the percentage difference for searches and sessions, and also the range of monthly differences found.

Some differences are striking. From March to May 2004, Proquest's Gender Watch search counts were reported as 30 percent lower with COUNTER while EBSCO's Health Source Nursing search counts were reported as 544 percent higher with COUNTER from October 2003 to February 2004. The extremes for sessions were a 28 percent decrease in reported session counts for Gender Watch and a 481 percent reported increase for EBSCO's Business Source Elite with the adoption of COUNTERcompliant statistics. Peter Shepherd, Project Director of COUNTER, has suggested that recorded use may decrease with the adoption of COUNTER practices, because the implementation of time filters eliminates multiple counting of requests and terms such as "successful request" are standardized. ${ }^{20}$ The data examined for this study showed, however, that not all electronic resources experienced a drop in search and session counts when moving from vendor-specific to COUNTER-compliant sta- 
tistics. Some resources, such as Proquest's Ethnic NewsWatch, did show a decrease in reported usage, but others, such as ERIC through EBSCO, reported increased use with the adoption of COUNTER. For some resources, such as Silver Platter's Social Work Abstracts, reported use stayed the same.

Table 2 illustrates change from month to month for searches and sessions respectively for a sample of resources chosen to illustrate the variation found in the data.

The data in Table 2 are subsets of the larger ranges in Table 1. Two Proquest databases, Ethnic NewsWatch and Gender Watch, had brief, three-month time periods in which COUNTER-compliant counts for both searches and sessions were initially reported as much lower than vendor-specific counts but ended up much higher than the vendor-specific statistics. The contrast between the two types of statistics each month is so striking that the authors speculate that COUNTER-compliant statistics actually began in mid-March and vendor-specific statistics end in mid-May. A third Proquest database with a different statistical interface, International Index to Music Periodicals, showed far less variation between the two forms of measurement for both searches and sessions over a longer time period. Elsevier ScienceDirect search statistics showed only a slight variation in searches. EBSCO databases showed large variations between vendor-specific and COUNTERcompliant statistics from month to month, from as little as a 31 percent difference in sessions (Health Source Consumer Edition, October 2003) to a 28,100 percent difference in sessions (MAS Ultra, January 2004); searches showed less extreme variations.

Tables 1 and 2 indicate that the adoption of COUNTER-compliant statistics may have a notable effect on the search and session data that a library receives from a vendor and point to the importance of librarians monitoring changes in the type of statistics received. Changes in reported use may reflect a different method of counting because of the adoption of COUNTER-compliant statistics rather than a difference in actual use of the resource.

COUNTER's codes of practice, for all of their advances toward standardization, allow for some differences in measurement among vendors or resources. COUNTER suggests, but does not mandate, a session timeout of thirty minutes. COUNTER requires that session timeouts of other than thirty minutes be noted in the audit. ${ }^{21}$ Vendors (and, in some cases, libraries) may choose different settings for timeouts, a function that affects session length and count of sessions. The authors asked six vendors of eleven resources about their timeout settings. One vendor set the timeout for one resource at five minutes; a second vendor set the timeout for one resource at ten minutes. A third vendor of three resources set the timeout at fifteen minutes for one resource and twenty minutes for the other two. The remaining three vendors set the timeouts for their six resources at the COUNTERrecommended default of thirty minutes. Two vendors also allowed the library to change the timeout period from the vendor's setting to a setting preferred by the library. The variations in timeout settings are, in effect, different rules for counting sessions. An electronic resource with a shorter timeout has a greater chance to record more sessions than a resource with a longer timeout. Thus, session statistics for electronic resources with different timeout settings should not be considered equivalent.

The COUNTER audit instructions also allow vendors to count searches differently. Appendix E of the COUNTER Code of Practice for Journals and Databases Release 2 states that "If a vendor's COUNTER reports do not include searches yielding zero results or when the number of results exceed some predefined threshold, then these categories of searches should be recorded separately and not included in the final tally." ${ }^{22}$ In short, although 


\begin{tabular}{|c|c|c|c|c|c|c|c|c|c|c|c|}
\hline \multirow{10}{*}{ 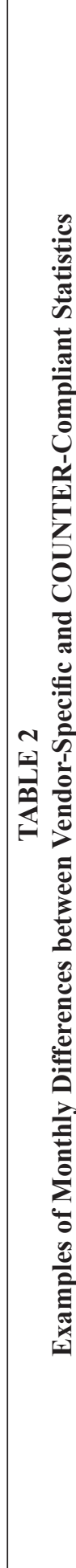 } & 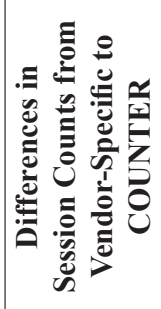 & 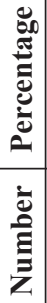 & $\begin{array}{l}\vec{\imath} \\
\vec{\imath}\end{array}$ & $\frac{n}{n}$ & $\hat{m}$ & $\stackrel{m}{T}$ & $\stackrel{n}{=}$ & $\begin{array}{l}8 \\
\infty \\
\infty \\
\text { d }\end{array}$ & ă & $\stackrel{\sim}{m}$ & $\begin{array}{l}5 \\
i \\
\text { in }\end{array}$ \\
\hline & 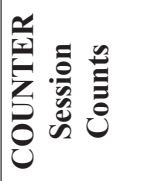 & & f & $\stackrel{\infty}{\sim}$ & $\stackrel{n}{\circ}$ & $r$ & ชิ & $\underset{\sim}{\infty}$ & in & $\frac{0}{m}$ & $\stackrel{0}{I}$ \\
\hline & 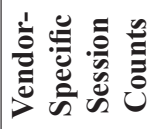 & & 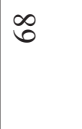 & $r$ & $\underset{0}{\infty}$ & ㄱ. & $\infty$ & - & $\sqrt[6]{6}$ & $n$ & $m$ \\
\hline & 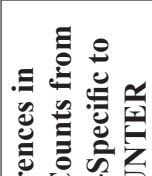 & 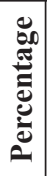 & p & $\begin{array}{l}\infty \\
\infty \\
n \\
n\end{array}$ & $\stackrel{\infty}{=}$ & nิ & ָ̃ & ণ & $\begin{array}{l}n \\
\infty \\
m\end{array}$ & రి & $\overleftrightarrow{Z}$ \\
\hline & 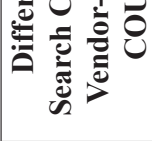 & 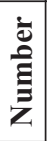 & to & $\frac{n}{f}$ & $\widehat{\widehat{\sigma}}$ & $\stackrel{0}{\sim}$ & ஓे & $\stackrel{N}{\stackrel{N}{N}}$ & 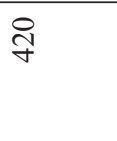 & $\hat{a}$ & $\stackrel{\infty}{\beth}$ \\
\hline & 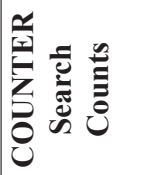 & & $\underset{\infty}{\infty}$ & $\vec{\delta}$ & g & $\vec{\nabla}$ & $\frac{\infty}{\sim}$ & $\underset{ల}{\mathbb{D}}$ & ๙ิ & $\begin{array}{l}2 \\
i n\end{array}$ & $\stackrel{\infty}{=}$ \\
\hline & 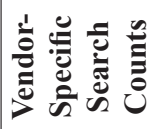 & & \& & 0 & $\frac{0}{m}$ & $n$ & $a$ & $\sigma$ & 8 & ชื & 0 \\
\hline & 兽 & & 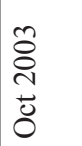 & 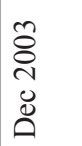 & 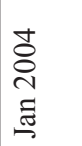 & 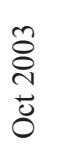 & 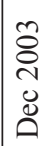 & 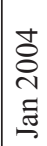 & 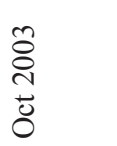 & $\begin{array}{l}\text { no } \\
\text { d } \\
\text { zo } \\
z\end{array}$ & 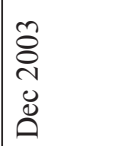 \\
\hline & 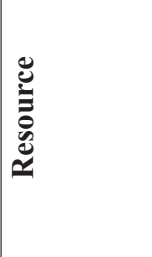 & & 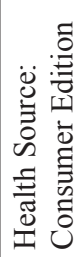 & 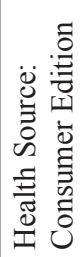 & 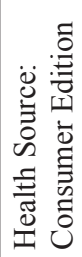 & 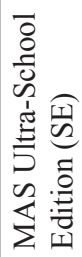 & 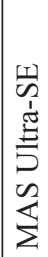 & 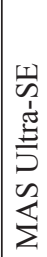 & 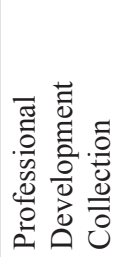 & 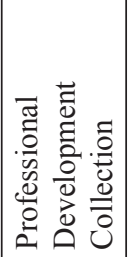 & 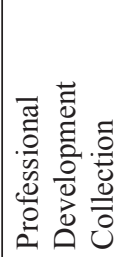 \\
\hline & $\frac{\dot{\vec{g}}}{\overline{0}}$ & & $\begin{array}{l}0 \\
\text { Un } \\
0 \\
\text { II }\end{array}$ & $\begin{array}{l}0 \\
0 \\
0 \\
0 \\
\text { II }\end{array}$ & $\begin{array}{l}0 \\
0 \\
0 \\
0 \\
I\end{array}$ & $\begin{array}{l}0 \\
\text { } \\
\mathscr{n} \\
\text { II }\end{array}$ & $\begin{array}{l}0 \\
0 \\
\infty \\
0 \\
1\end{array}$ & $\begin{array}{l}0 \\
\text { e } \\
0 \\
\text { II }\end{array}$ & 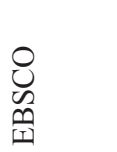 & $\begin{array}{l}0 \\
0 \\
\infty \\
01\end{array}$ & $\begin{array}{l}0 \\
0 \\
\infty \\
0 \\
1\end{array}$ \\
\hline
\end{tabular}




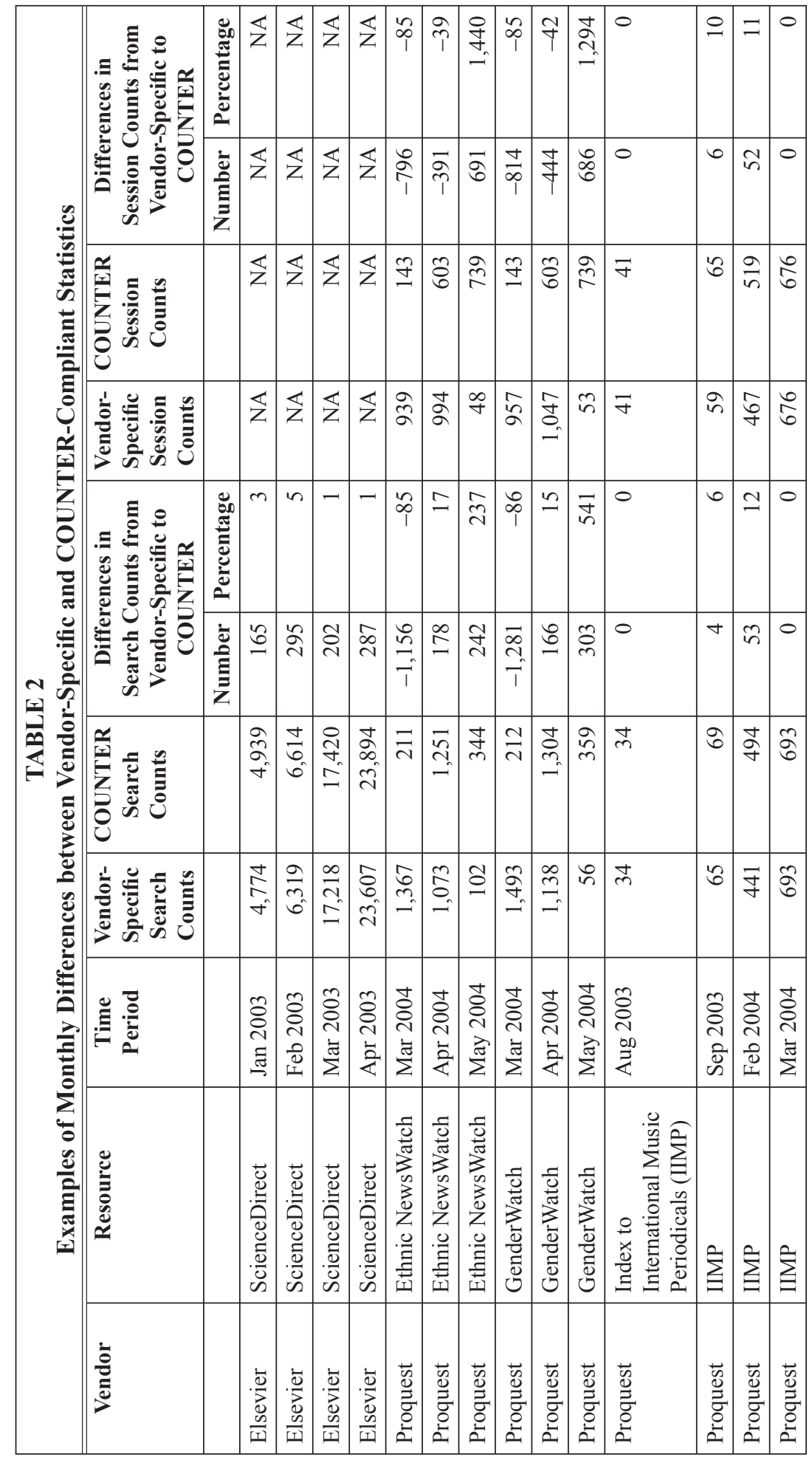


COUNTER standardizes practices for measuring and reporting use of electronic resources and provides for auditing vendor counts, COUNTER permits vendors some variation for counting searches and sessions.

The migration to COUNTER-compliant statistics and the evolution of COUNTER will continue. To interpret both vendorspecific and COUNTER-compliant use statistics in a meaningful manner, it is important for librarians to be aware that changes in data-gathering mechanisms may affect vendor-reported use data and that vendors may vary their timeout settings and search thresholds and thus affect search and session counts.

While this article explores searches and sessions, complications in interpretation of use data are not limited to these two measures. Phillip M. Davis and Jason S. Price found that interface design affects counts of items in COUNTER-compliant reports. Full text downloads are greater when publishers place HTML between users and PDF versions and when linking mechanisms go to the full text, not the abstract. $^{23}$

NISO (National Information Standards Organization) is sponsoring the Standardized Usage Statistics Harvesting Initiative (SUSHI), an effort to design a standard model for automated statistics gathering. ${ }^{24}$ Private sector development of tools for gathering usage data, such as ScholarlyStats by MPS Technologies and Thomson's Journal Use Reports will complement this initiative. ${ }^{25,26}$ Whether such efforts, principally aimed at gathering use data rather than determining use measures, will also lead to greater standardization in vendor-reported counts of use remains to be seen.

\section{Search Alert Services}

Search alerts are stored searches that are run in a resource automatically by the electronic resource's search engine itself at regular intervals. Several vendors offer search alert services to patrons, and those vendors differ in the ways they count search alerts in usage statistics. Table 3 summarizes vendor practices for counting search alerts in use statistics, practices that were confirmed in fall 2004 via e-mail correspondence with the vendor.

In most cases, a search alert is not counted as a search or a session. However, the authors learned that Cambridge Scientific Abstracts did count a search alert as a search and a session in the statistics it reported. Proquest counted running an alert as a search and gave the library a choice as to whether or not the alert would count as a session. Usage statistics from Elsevier's ScienceDirect and Thomson ISI reported alerts as separate counts each time a stored alert ran but did not count the running of an alert as a search or session.

When an alert feature runs automatic updates, it has the potential to both help users get the most current information and increase the reported usage of the database. The more alerts that users set up, the greater the potential impact of alerts on the reported statistics. Yet, the differences among methods of counting search alerts by vendors means that the impact of search alerts will vary by database, and those differences should be considered when reviewing use statistics.

The authors also found a variety of other alerts available from vendors, such as table of contents alerts, topic alerts, and citation alerts. These types of alerts were less frequently available than search alerts, but they may have an impact on use statistics as well. The advent of RSS feeds, with many types of alerting possibilities, may have a much larger impact on electronic resource use than search alert services, and it will be important for librarians to understand how RSS feed use will be recorded in use statistics.

\section{Federated Searching}

Federated searching is the use of a search engine to send one search to multiple databases within or across vendor platforms. The study library began using a federated search engine, WebFeat, in March 2001, 


\begin{tabular}{|c|c|c|c|}
\hline \multicolumn{4}{|c|}{$\begin{array}{c}\text { TABLE } 3 \\
\text { Vendor Practices for Counting Search Alerts in Use Statistics }\end{array}$} \\
\hline Vendor & $\begin{array}{l}\text { Does the running } \\
\text { of the search alert } \\
\text { count as a search? }\end{array}$ & $\begin{array}{l}\text { Does the running } \\
\text { of the search alert } \\
\text { count as a session? }\end{array}$ & $\begin{array}{l}\text { Are search alerts } \\
\text { reported as a } \\
\text { separate count? }\end{array}$ \\
\hline ABC-Clio & No & No & No \\
\hline $\begin{array}{l}\text { Cambridge Scientific } \\
\text { Abstracts }\end{array}$ & Yes & Yes & No \\
\hline EBSCO & No & No & No \\
\hline $\begin{array}{l}\text { Elsevier } \\
\text { ScienceDirect }\end{array}$ & No & No & Yes \\
\hline Ingenta & No & No & No \\
\hline Ovid & No & No & No \\
\hline Project Muse & No & No & No \\
\hline Proquest & Yes & $\begin{array}{l}\text { A library can choose } \\
\text { to count or not }\end{array}$ & No \\
\hline Thomson ISI & No & No & Yes \\
\hline
\end{tabular}

while serving as a development partner. With WebFeat, the study library's users can search, with one search statement, all of the more than 100 databases available via WebFeat, one or more broad subject categories (e.g., arts and humanities- 43 databases; health sciences- 44 databases as of November 2005), or any combination of individual databases. Because of the terms of the study library's contract with WebFeat and the configuration of some of its electronic resources, not all of them were searchable using WebFeat during the study's time frame. WebFeat restricts access to users affiliated with the study library, and each database that WebFeat searches records use in the statistics that the database's vendor provides. The authors observed a difference between usage statistics from databases searchable via WebFeat and databases that were not searchable via WebFeat. Counts of searches and sessions and the ratios of searches to sessions reveal this difference.

Blecic, Fiscella, and Wiberley reported that the ratio of searches per session can be useful to monitor change or trends because it tends to be fairly stable over time unless something differs in the way use data are collected or in the way a resource is being used. ${ }^{27}$ Federated searching changes the way electronic resources are used, because users can search many resources at one time. Each federated search via WebFeat generates one session and one search per electronic resource searched. Testing by the authors indicates that, if a user searches through WebFeat two or more times within a short span, each search counts as a new search and session in the searched databases' use statistics rather than a second search within one session. The authors of the present study hypothesized that, for resources searchable via WebFeat, the searches per session ratio would move toward one, as use of WebFeat generated many sessions with only one search each. The authors also hypothesized that for those resources not searchable via WebFeat, the searches per session ratio would remain fairly stable because there was no influx of sessions with just one search.

Table 4 shows three years of use statistics for thirty-one resources that were searchable via WebFeat since its inception in March 2001 at the study library. During the time period studied, these resources have been consistent in content and in 


\begin{tabular}{|c|c|c|c|c|c|c|}
\hline \multicolumn{7}{|c|}{$\begin{array}{c}\text { TABLE } 4 \\
\text { Trends in Searches and Sessions for Databases Searchable via WebFeat }\end{array}$} \\
\hline Resource/Vendor & $\begin{array}{c}\text { Fiscal } \\
\text { Year } \\
\text { (July 1- } \\
\text { June 30) }\end{array}$ & Searches & Sessions & $\begin{array}{l}\text { Percentage } \\
\text { Increase in } \\
\text { Searches } \\
\text { from } \\
\text { FY2002 to } \\
\text { FY2004 }\end{array}$ & $\begin{array}{c}\text { Searches } \\
\text { per } \\
\text { Session }\end{array}$ & $\begin{array}{c}\text { Trend of } \\
\text { Searches per } \\
\text { Session }\end{array}$ \\
\hline \multirow{3}{*}{$\begin{array}{l}\text { ABI Inform/ } \\
\text { OCLC }\end{array}$} & 2002 & 13,693 & 6,328 & & 2.164 & \multirow{3}{*}{$\begin{array}{l}\text { Down } \\
\text { Toward } 1\end{array}$} \\
\hline & 2003 & 13,754 & 7,031 & & 1.956 & \\
\hline & 2004 & 17,889 & 13,184 & 31 & 1.357 & \\
\hline \multirow{3}{*}{$\begin{array}{l}\text { African } \\
\text { American } \\
\text { Biographical } \\
\text { Database/ } \\
\text { Proquest }\end{array}$} & 2002 & 112 & 34 & & 3.294 & \multirow[b]{3}{*}{$\begin{array}{l}\text { Down } \\
\text { Below } 1\end{array}$} \\
\hline & 2003 & 1,095 & 744 & & 1.472 & \\
\hline & 2004 & 6,603 & 6,749 & 5,796 & 0.978 & \\
\hline \multirow{3}{*}{$\begin{array}{l}\text { America: History } \\
\text { and Life/ABC- } \\
\text { Clio }\end{array}$} & 2002 & 2,966 & 1,391 & & 2.132 & \multirow{3}{*}{$\begin{array}{l}\text { Down } \\
\text { Below } 1\end{array}$} \\
\hline & 2003 & 3,819 & 2,257 & & 1.692 & \\
\hline & 2004 & 6,566 & 8,441 & 121 & 0.679 & \\
\hline \multirow{3}{*}{$\begin{array}{l}\text { Article First/ } \\
\text { OCLC }\end{array}$} & 2002 & 34,012 & 21,231 & & 1.602 & \multirow[b]{3}{*}{ Up } \\
\hline & 2003 & 42,687 & 26,052 & & 1.639 & \\
\hline & 2004 & 61,608 & 37,053 & 81 & 1.663 & \\
\hline \multirow{3}{*}{$\begin{array}{l}\text { ATLA Religion/ } \\
\text { OCLC }\end{array}$} & 2002 & 1,482 & 806 & & 1.839 & \multirow{3}{*}{$\begin{array}{l}\text { Down } \\
\text { Below } 1\end{array}$} \\
\hline & 2003 & 2,433 & 1,748 & & 1.392 & \\
\hline & 2004 & 6,616 & 6,892 & 346 & 0.96 & \\
\hline \multirow{3}{*}{$\begin{array}{l}\text { Books in Print/ } \\
\text { OCLC }\end{array}$} & 2002 & 3,226 & 1,458 & & 2.213 & \multirow{3}{*}{$\begin{array}{l}\text { Down } \\
\text { Toward } 1\end{array}$} \\
\hline & 2003 & 3,679 & 1,965 & & 1.872 & \\
\hline & 2004 & 9,267 & 7,084 & 187 & 1.308 & \\
\hline \multirow{3}{*}{$\begin{array}{l}\text { Business \& } \\
\text { Company } \\
\text { Resource Center/ } \\
\text { Thomson Gale }\end{array}$} & 2002 & 15,217 & 2,021 & & 7.529 & \multirow{3}{*}{$\begin{array}{l}\text { Down } \\
\text { Toward } 1\end{array}$} \\
\hline & 2003 & 15,268 & 2,543 & & 6.004 & \\
\hline & 2004 & 49,090 & 8,601 & 223 & 5.707 & \\
\hline \multirow{3}{*}{$\begin{array}{l}\text { Contemporary } \\
\text { Women's Issues/ } \\
\text { OCLC }\end{array}$} & 2002 & 3,511 & 3,125 & & 1.124 & \multirow{3}{*}{$\begin{array}{l}\text { Mixed } \\
\text { Down Overall } \\
\text { Below } 1\end{array}$} \\
\hline & 2003 & 4,875 & 4,186 & & 1.165 & \\
\hline & 2004 & 8,305 & 9,132 & 137 & 0.909 & \\
\hline \multirow{3}{*}{$\begin{array}{l}\text { EBM Reviews/ } \\
\text { OVID }\end{array}$} & 2002 & 16,682 & 4,150 & & 4.020 & \multirow{3}{*}{$\begin{array}{l}\text { Mixed } \\
\text { Down Overall } \\
\text { Toward } 1\end{array}$} \\
\hline & 2003 & 17,064 & 4,195 & & 4.068 & \\
\hline & 2004 & 11,933 & 3,575 & -28 & 3.338 & \\
\hline \multirow[t]{3}{*}{ Econlit/OCLC } & 2002 & 5,441 & 2,468 & & 2.205 & \multirow{3}{*}{$\begin{array}{l}\text { Down } \\
\text { Toward } 1\end{array}$} \\
\hline & 2003 & 6,670 & 3,509 & & 1.901 & \\
\hline & 2004 & 10,892 & 8,902 & 100 & 1.224 & \\
\hline \multirow{3}{*}{$\begin{array}{l}\text { Ethnic } \\
\text { NewsWatch/ } \\
\text { Proquest }\end{array}$} & 2002 & 2,427 & 1,055 & & 2.3 & \multirow{3}{*}{$\begin{array}{l}\text { Down } \\
\text { Toward } 1\end{array}$} \\
\hline & 2003 & 4,653 & 2,505 & & 1.857 & \\
\hline & 2004 & 8,061 & 6,507 & 232 & 1.239 & \\
\hline
\end{tabular}




\begin{tabular}{|c|c|c|c|c|c|c|}
\hline \multicolumn{7}{|c|}{$\begin{array}{c}\text { TABLE } 4 \\
\text { Trends in Searches and Sessions for Databases Searchable via WebFeat }\end{array}$} \\
\hline Resource/Vendor & $\begin{array}{c}\text { Fiscal } \\
\text { Year } \\
\text { (July 1- } \\
\text { June 30) }\end{array}$ & Searches & Sessions & $\begin{array}{c}\text { Percentage } \\
\text { Increase in } \\
\text { Searches } \\
\text { from } \\
\text { FY2002 to } \\
\text { FY2004 } \\
\end{array}$ & $\begin{array}{l}\text { Searches } \\
\text { per } \\
\text { Session }\end{array}$ & $\begin{array}{c}\text { Trend of } \\
\text { Searches per } \\
\text { Session }\end{array}$ \\
\hline \multirow{3}{*}{$\begin{array}{l}\text { GenderWatch/ } \\
\text { Proquest }\end{array}$} & 2002 & 2,280 & 780 & & 2.923 & \multirow{3}{*}{$\begin{array}{l}\text { Down } \\
\text { Toward } 1\end{array}$} \\
\hline & 2003 & 5,201 & 2,032 & & 2.56 & \\
\hline & 2004 & 8,602 & 6,035 & 277 & 1.425 & \\
\hline \multirow{3}{*}{$\begin{array}{l}\text { General } \\
\text { Business, } \\
\text { with Backfile/ } \\
\text { Thomson Gale }\end{array}$} & 2002 & 3,618 & 1,314 & & 2.753 & \multirow{3}{*}{$\begin{array}{l}\text { Down } \\
\text { Toward } 1\end{array}$} \\
\hline & 2003 & 2,983 & 1,335 & & 2.234 & \\
\hline & 2004 & 4,387 & 2,202 & 21 & 1.992 & \\
\hline \multirow{3}{*}{$\begin{array}{l}\text { GPO Monthly } \\
\text { Catalog/OCLC }\end{array}$} & 2002 & 366 & 343 & & 1.067 & \multirow{3}{*}{$\begin{array}{l}\text { Down } \\
\text { Below } 1\end{array}$} \\
\hline & 2003 & 1,023 & 1,114 & & 0.918 & \\
\hline & 2004 & 3,862 & 4,979 & 955 & 0.776 & \\
\hline \multirow{3}{*}{$\begin{array}{l}\text { Health and } \\
\text { Psychosocial } \\
\text { Instruments/ } \\
\text { OVID }\end{array}$} & 2002 & 5,245 & 857 & & 6.12 & \multirow{3}{*}{$\begin{array}{l}\text { Down } \\
\text { Toward } 1\end{array}$} \\
\hline & 2003 & 3,440 & 853 & & 4.033 & \\
\hline & 2004 & 6,586 & 5,245 & 26 & 1.256 & \\
\hline \multirow{3}{*}{$\begin{array}{l}\text { Historical } \\
\text { Abstracts/ABC- } \\
\text { Clio }\end{array}$} & 2002 & 2,218 & 1,113 & & 1.993 & \multirow{3}{*}{$\begin{array}{l}\text { Down } \\
\text { Below } 1\end{array}$} \\
\hline & 2003 & 2,102 & 1,392 & & 1.51 & \\
\hline & 2004 & 4,327 & 6,371 & 95 & 0.679 & \\
\hline \multirow{3}{*}{$\begin{array}{l}\text { International } \\
\text { Index to Music } \\
\text { Periodicals/ } \\
\text { Proquest }\end{array}$} & 2002 & 641 & 193 & & 3.321 & \multirow{3}{*}{$\begin{array}{l}\text { Down } \\
\text { Below } 1\end{array}$} \\
\hline & 2003 & 1,030 & 331 & & 3.112 & \\
\hline & 2004 & 5,002 & 5,042 & 680 & 0.992 & \\
\hline \multirow{3}{*}{$\begin{array}{l}\text { International } \\
\text { Pharmaceutical } \\
\text { Abstracts/OVID }\end{array}$} & 2002 & 3,896 & 964 & & 4.041 & \multirow{3}{*}{$\begin{array}{l}\text { Down } \\
\text { Toward } 1\end{array}$} \\
\hline & 2003 & 1,403 & 367 & & 3.823 & \\
\hline & 2004 & 5,027 & 4,377 & 29 & 1.149 & \\
\hline \multirow[t]{3}{*}{ Medline/OCLC } & 2002 & 10,473 & 5,039 & & 2.078 & \multirow{3}{*}{$\begin{array}{l}\text { Down } \\
\text { Toward } 1\end{array}$} \\
\hline & 2003 & 10,177 & 5,003 & & 2.034 & \\
\hline & 2004 & 11,179 & 7,254 & 7 & 1.541 & \\
\hline \multirow{3}{*}{$\begin{array}{l}\text { OCLC ECO/ } \\
\text { OCLC }\end{array}$} & 2002 & 120,842 & 96,026 & & 1.258 & \multirow[b]{3}{*}{ Up } \\
\hline & 2003 & 154,617 & 97,456 & & 1.587 & \\
\hline & 2004 & 174,034 & 108,615 & 44 & 1.602 & \\
\hline \multirow{3}{*}{$\begin{array}{l}\text { Papersfirst/ } \\
\text { OCLC }\end{array}$} & 2002 & 1,603 & 1,561 & & 1.027 & \multirow{3}{*}{$\begin{array}{l}\text { Down } \\
\text { Below } 1\end{array}$} \\
\hline & 2003 & 2,281 & 2,352 & & 0.97 & \\
\hline & 2004 & 5,483 & 6,159 & 242 & 0.89 & \\
\hline \multirow{3}{*}{$\begin{array}{l}\text { Periodicals } \\
\text { Abstracts/OCLC }\end{array}$} & 2002 & 12,540 & 8,292 & & 1.512 & \multirow{3}{*}{$\begin{array}{l}\text { Down } \\
\text { Toward } 1\end{array}$} \\
\hline & 2003 & 14,244 & 9,692 & & 1.47 & \\
\hline & 2004 & 14,657 & 10,907 & 17 & 1.344 & \\
\hline
\end{tabular}




\begin{tabular}{|c|c|c|c|c|c|c|}
\hline \multicolumn{7}{|c|}{$\begin{array}{c}\text { TABLE } 4 \\
\text { Trends in Searches and Sessions for Databases Searchable via WebFeat }\end{array}$} \\
\hline Resource/Vendor & $\begin{array}{c}\text { Fiscal } \\
\text { Year } \\
\text { (July 1- } \\
\text { June 30) }\end{array}$ & Searches & Sessions & $\begin{array}{c}\text { Percentage } \\
\text { Increase in } \\
\text { Searches } \\
\text { from } \\
\text { FY2002 to } \\
\text { FY2004 }\end{array}$ & $\begin{array}{c}\text { Searches } \\
\text { per } \\
\text { Session }\end{array}$ & $\begin{array}{c}\text { Trend of } \\
\text { Searches per } \\
\text { Session }\end{array}$ \\
\hline \multirow{3}{*}{$\begin{array}{l}\text { Proceedings } \\
\text { First/OCLC }\end{array}$} & 2002 & 390 & 452 & & 0.863 & \multirow{3}{*}{$\begin{array}{l}\text { Down } \\
\text { Below } 1\end{array}$} \\
\hline & 2003 & 879 & 1,186 & & 0.741 & \\
\hline & 2004 & 3,226 & 5,275 & 727 & 0.612 & \\
\hline \multirow{3}{*}{$\begin{array}{l}\text { Project Muse/ } \\
\text { JHU Press } \\
\text { with Milton } \\
\text { S. Eisenhower } \\
\text { Library }\end{array}$} & 2002 & 1,351 & 3,128 & & 0.432 & \multirow[b]{3}{*}{ Up } \\
\hline & 2003 & 3,181 & 3,533 & & 0.9 & \\
\hline & 2004 & 6,633 & 4,689 & 391 & 1.415 & \\
\hline \multirow{3}{*}{$\begin{array}{l}\text { PROMT/ } \\
\text { Thomson Gale }\end{array}$} & 2002 & 1,131 & 444 & & 2.547 & \multirow{3}{*}{$\begin{array}{l}\text { Mixed } \\
\text { Down Overall } \\
\text { Toward } 1 \\
\end{array}$} \\
\hline & 2003 & 767 & 1,319 & & 0.582 & \\
\hline & 2004 & 879 & 524 & -22 & 1.677 & \\
\hline \multirow[t]{3}{*}{ RILM/OCLC } & 2002 & 399 & 328 & & 1.216 & \multirow{3}{*}{$\begin{array}{l}\text { Down } \\
\text { Below } 1\end{array}$} \\
\hline & 2003 & 845 & 896 & & 0.943 & \\
\hline & 2004 & 2,177 & 2,761 & 446 & 0.788 & \\
\hline \multirow{3}{*}{$\begin{array}{l}\text { Union Lists/ } \\
\text { OCLC }\end{array}$} & 2002 & 508 & 478 & & 1.063 & \multirow{3}{*}{$\begin{array}{l}\text { Down } \\
\text { Below } 1\end{array}$} \\
\hline & 2003 & 936 & 1,128 & & 0.83 & \\
\hline & 2004 & 2,291 & 3,161 & 351 & 0.725 & \\
\hline \multirow{3}{*}{$\begin{array}{l}\text { Web of Science/ } \\
\text { Thomson( } 2003 \\
\text { data incomplete) }\end{array}$} & 2002 & 94,665 & 25,797 & & 3.67 & \multirow{3}{*}{$\begin{array}{l}\text { Mixed } \\
\text { Down Overall } \\
\text { Toward } 1\end{array}$} \\
\hline & 2003 & 58,279 & 12,965 & & 4.495 & \\
\hline & 2004 & 143,614 & 49,272 & 52 & 2.915 & \\
\hline \multirow{3}{*}{$\begin{array}{l}\text { Wilson Select } \\
\text { Plus/OCLC }\end{array}$} & 2002 & 36,595 & 22,196 & & 1.649 & \multirow{3}{*}{$\begin{array}{l}\text { Mixed } \\
\text { Down Overall } \\
\text { Toward } 1\end{array}$} \\
\hline & 2003 & 39,646 & 25,324 & & 1.566 & \\
\hline & 2004 & 36,266 & 22,789 & -1 & 1.591 & \\
\hline \multirow{3}{*}{$\begin{array}{l}\text { World Almanac/ } \\
\text { OCLC }\end{array}$} & 2002 & 230 & 313 & & 0.735 & \multirow{3}{*}{$\begin{array}{l}\text { Mixed } \\
\text { Up Overall }\end{array}$} \\
\hline & 2003 & 1,383 & 1,596 & & 0.867 & \\
\hline & 2004 & 2,619 & 3,294 & 104 & 0.795 & \\
\hline \multirow[t]{3}{*}{ WorldCat/OCLC } & 2002 & 66,139 & 26,539 & & 2.492 & \multirow{3}{*}{$\begin{array}{l}\text { Down } \\
\text { Toward } 1\end{array}$} \\
\hline & 2003 & 78,250 & 31,791 & & 2.461 & \\
\hline & 2004 & 74,530 & 33,077 & 13 & 2.253 & \\
\hline
\end{tabular}

the way the vendors reported usage. Of the thirty-one resources, twenty-seven showed a decrease in the searches per session ratio from fiscal year 2002 to 2004 . Of these twenty-seven resources, sixteen showed a trend downward toward a ratio of one query per session. The other eleven showed the ratio moving below one, in which there was less than one search per session in fiscal year 2004.

While federated searching was introduced in March 2001, full implementation 
was gradual as translators for various databases were refined during the development phase. The study library delayed promotion and teaching of WebFeat during development, although the resource was available to the library's users. The improvement of translators and delayed promotion may explain why the trends toward one search per session became more pronounced during FY2002-FY2004. At the same time that the searches per session ratio declined for most of the resources searchable using WebFeat, the overall use of these resources shows some precipitous increases. Except for three resources, all show an increase in searches between FY2002 and FY2004, and in most cases a large leap between FY2003 and FY2004.

Table 5 shows the use data and searches per session ratios for six databases that were not searchable via WebFeat as of June 30, 2004.

These databases show different searches per session patterns from those searchable using WebFeat. All but one showed an increase in the searches per session ratio from FY2002 to FY2004. Growth in the number of searches from FY2002 to FY2004 was more modest than the gains observed in Table 4. Resources not searchable via WebFeat displayed different use patterns from those searchable via WebFeat.

While the authors suspect that federated searching contributed to the rise in sessions and searches for databases that WebFeat searches, they realize that other influences, such as changes in user preferences, library instruction, course management software, electronic reserves, and class assignments, may have helped increase use. Another factor that may have had an effect was open URL technology for linking among databases, which was implemented at the study library beginning in March 2003. Open URL linking enables a user who clicks on a citation in one resource to go directly to the cited item, which may be in another resource. Since linking does not involve formulating an intellectual query when entering a database, a link-in would most likely produce just one session count with no search in that database's use statistics. Therefore, the implementation of open URL linking could also have contributed to the decline in the search per session ratio seen in Table 4 and perhaps the overall rise in use as navigation between databases became easier. However, seven of the databases that were not part of open URL linking but searchable via WebFeat (those from Proquest, Thomson Gale, and Project Muse) showed a decrease in the search per session ratio, while one showed an increase. This argues that federated searching, independent of open URL linking technology, had an impact on electronic resource use.

Overall, the authors found that rapidly changing technology is having a noticeable effect on the way in which electronic resources are used. The potential for different innovations to interact increases the complexity of interpretation of use statistics. As electronic use patterns change noticeably over time, librarians need to explore these changes in relationship to the changes in functionality of electronic resources in their library. Careful record keeping of implementation of innovation and communication among those implementing new functionality, vendors producing use statistics reports, and those analyzing the reports are necessary for meaningful interpretation of use statistics.

\section{Conclusion}

The ICOLC guidelines and Project COUNTER have greatly improved vendor-supplied statistics about use of electronic resources. ICOLC established measures of use, and COUNTER standardizes the way vendors count and report use data. The COUNTER reports make it easier to collect data, and standardization is leading to the development of protocols, such as SUSHI, and third-party services, such as ScholarlyStats and Journal Use Reports, to collect use statistics across 


\begin{tabular}{|c|c|c|c|c|c|c|}
\hline Trends in Sear & hes and & Sessions & $\begin{array}{l}\text { ГABLE } \\
\text { or Datal }\end{array}$ & ases Not $\mathrm{S}$ & archable & via WebFeat \\
\hline Resource/Vendor & $\begin{array}{c}\text { Fiscal } \\
\text { Year } \\
\text { (July 1- } \\
\text { June 30) }\end{array}$ & Searches & Sessions & $\begin{array}{c}\text { Percentage } \\
\text { Increase in } \\
\text { Searches } \\
\text { from } \\
\text { FY2002 to } \\
\text { FY2004 }\end{array}$ & $\begin{array}{l}\text { Searches } \\
\text { per } \\
\text { Session }\end{array}$ & $\begin{array}{l}\text { Trend of } \\
\text { Searches per } \\
\text { Session }\end{array}$ \\
\hline Archives USA/ & 2002 & 701 & 203 & & 3.453 & Mixed Down \\
\hline Proquest & 2003 & 945 & 209 & & 4.522 & Overall \\
\hline & 2004 & 841 & 450 & 20 & 1.869 & Toward 1 \\
\hline Beilstein & 2002 & 29,610 & 8,446 & & 3.506 & \\
\hline Gmelin/MDL & 2003 & 31,465 & 8,226 & & 3.825 & \\
\hline Systems & 2004 & 31,061 & 7,397 & 5 & 4.199 & Up \\
\hline Early English & 2002 & 361 & 112 & & 3.223 & \\
\hline Books Online/ & 2003 & 299 & 130 & & 2.3 & Mixed \\
\hline & 2004 & 1,031 & 298 & 186 & 3.460 & Up Overall \\
\hline NASW & 2002 & 837 & 418 & & 2.002 & \\
\hline Directory of & 2003 & 789 & 330 & & 2.391 & \\
\hline $\begin{array}{l}\text { Workers/ } \\
\text { SilverPlatter }\end{array}$ & 2004 & 1,587 & 474 & 90 & 3.348 & Up \\
\hline Philosopher's & 2002 & 3,257 & 901 & & 3.615 & \\
\hline Index/ & 2003 & 2,494 & 798 & & 3.126 & Mixed \\
\hline & 2004 & 3,732 & 1,020 & 15 & 3.659 & Up Overall \\
\hline Social Work & 2002 & 5,607 & 1,557 & & 3.601 & \\
\hline Abstracts/ & 2003 & 4,268 & 1,250 & & 3.414 & Mixed \\
\hline & 2004 & 5,994 & 1,458 & 7 & 4.111 & Up Overall \\
\hline
\end{tabular}

vendors. By auditing vendor statistics, COUNTER removes a potential source of doubt about reliability of vendor reports. At the same time, the variation allowed by COUNTER in counting of sessions and searches means that data across vendors may not be counted in the same way. More significant, increased functionality of electronic resources and systems complicate interpretation of two key measures in COUNTER - sessions and searches.

One can argue that, over time, the meanings of sessions and searches have been changing. In the first years of electronic resources, users might mistakenly start a session as a result of a keying or clicking error or because they thought a database had subject coverage it did not (e.g., thinking that Historical Abstracts covered American history). Still, most sessions probably began because users consciously and correctly chose to access a given database. In the moments between starting a session and searching, users had a chance to confirm that they had accessed the database they sought. Thus, searches even more than sessions were a measure of users' conscious and deliberate preferences. Today, with federated searching, a session and a search do not have the same meaning. Sessions and searches generated through federated searching are more likely scatter shots across one or more groups of databases rather than 
a deliberate choice of an individual database. The more users access and search a database through federated searching, the less meaningful are counts of sessions and searches of that database.

The meanings of sessions and searches also change as more databases provide alert services and as more users set up alerts. On the one hand, because most alerts run automatically, one can argue that, as with federated searching, the alert-generated sessions and searches that vendors add to totals of sessions and searches are not the product of individual choices and are less meaningful. On the other hand, one can argue that, because users have taken the trouble to set up an alert and want the regular reports that alert generates, that database is especially valuable to them and running of alerts should be counted because the count reflects that value. This argument is moot for the vendors that do not count the running of alerts in use statistics.

Open URL linking technology, RSS feeds, and further advancements in functionality will continue to change how patrons use electronic resources and will affect the meaning of searches and sessions. The findings of this study suggest the following practical steps that librarians should take when working with vendor-reported measures of use of electronic resources:

- Monitor changes in how vendors report statistics, especially when a vendor switches from vendor-specific to COUNTER-compliant statistics. If possible, compare vendor-specific and COUNTERcompliant data for the same time period. Rises or falls in counts may be as much an artifact of the types of statistics as changes in use. Monitor further developments that may occur with the implementation of SUSHI and commercial statistics gathering products.

- When comparing statistics for different resources, ascertain the timeout settings for each resource, the search result parameters that the vendor uses for counts of searches (e.g., searches that re- trieve no results do not count as searches), and whether sessions are counted if they do not have a search. As open URL linking becomes more widespread, whether vendors count link-ins as sessions or a separate statistical category becomes a more important question.

- Identify databases that offer alert services and learn how those databases count the running of alerts, whether included with regular counts of sessions and searches, counted separately or not counted at all. Variations in the way vendors count the running of alerts affects the comparability of data for various resources. Monitor the effects of new alerting technology such as RSS feeds on electronic resources use statistics.

- Consider the potential effects of federated searching and other enhancements in electronic resource functionality when interpreting use statistics. The results of this study offer evidence that the introduction of a federated search engine may alter the use of electronic resources over time, arguably increasing overall use while changing patterns of searching individual resources. Open URL linking technology may also affect use as may any future innovations in functionality of electronic resources.

It is almost trite to say that the future will bring innovations in functionality within and among electronic resources. But trite as it may sound, innovation in resource functionality is probably the central issue for librarians who need meaningful measures of electronic resources. The analysis in this article suggests that innovations in functionality may have changed the meaning of sessions and searches. The analysis also suggests the following principle: innovations in electronic resource functionality will necessitate advances in electronic resource usage measures to describe use meaningfully.

The ICOLC guidelines and Project COUNTER have improved measures of use and the reporting of those measures. If these better methods of counting and re- 
porting searches and sessions do not meet librarians' needs because innovations in electronic resource functionality have made those measures less meaningful, then the field will refine the ways these measures are derived. Alternatively, it may be that other measures will become more important; for example, perhaps counts of items accessed will become the most telling measure of use. In any case, librarians will need to monitor innovations in the electronic resource environment and advances in use statistics to understand what vendors report.

\section{Notes}

1. Mary M. Case, "A Snapshot in Time: ARL Libraries and Electronic Journal

Resources," ARL, no. 235 (Aug. 2004): 1-10. Available online at http://www.arl.org/newsltr/235/ snapshot.html. [Accessed 13 February 2006].

2. Martha Kyrillidou and Mark Young, ARL Statistics, 2003-2004 (Washington, D.C.: ARL, 2005), p. 88. Available online at http://www.arl.org/stats/pubpdf/arlstat04.pdf. [Accessed 13 February 2006].

3. ARL Supplementary Statistics 2003-2004 (questionnaire). Available online at http://www. arl.org/stats/mailing/m04/04ssurvey.pdf. [Accessed 13 February 2006].

4. James Pringle, "Secondary Publishers and Usage Statistics," Online Usage Statistics: A Publisher's Guide, ed. Bernard Rous (New York: Association of American Publishers, 2004), 73-90.

5. International Coalition of Library Consortia, "Guidelines for Statistical Measures of Usage of Web-Based Indexed, Abstracted, and Full-Text Resources," Nov. 1998. Available online at http://www.library.yale.edu/consortia/webstats.html. [Accessed 13 February 2006].

6. International Coalition of Library Consortia, "Guidelines for Statistical Measures of Usage of Web-Based Information Resources," Dec. 2001 revision. Available online at http://www.library. yale.edu/consortia/2001webstats.htm. [Accessed 13 February 2006].

7. COUNTER, Code of Practice for Journals and Databases, Release 1, December 2002. Available online at http://www.projectcounter.org/code_practice_r1.html. [Accessed 13 February 2006].

8. COUNTER, Code of Practice for Journals and Databases, Release 2, Appendix

A, Glossary of Terms. 3.1.4.2 "Session" April 2005. Available online at http://www.projectcounter.org/r2/R2_Appendix_A_Glossary.pdf. [Accessed 13 February 2006].

9. COUNTER, Code of Practice for Journals and Databases, Release 2, Appendix

E, Auditing Requirements and Tests. April 2005. Available online at http://www.projectcounter. org/r2/R2_Appendix_E_Auditing_Requirements_and_Tests.pdf. [Accessed 13 February 2006].

10. International Coalition of Library Consortia, "Guidelines," December 2001, p. 1.

11. COUNTER, Code of Practice for Journals and Databases, Release 2, Appendix A, 3.1.2.10 "Search."

12. International Coalition of Library Consortia. "Guidelines," December 2001, p. 1-2.

13. Tim Ingoldsby, "Usage Statistics for the System Administrator," in Online Usage Statistics, ed. Rous, 17-23.

14. Wonsik Shim, Kurt Murphy, and Dennis Brunning, "Usage Statistics for Electronic Services and Resources: A Library Perspective," in Online Usage Statistics, ed. Rous, 34-46.

15. Oliver Pesch, "Usage Statistics: The Full Text Aggregator," in Online Usage Statistics, ed. Rous, 91-112.

16. COUNTER, The COUNTER Code of Practice for Journals and Databases, Release 2, Section 2.3. April 2005. Available online at http://www.projectcounter.org/r2/COUNTER_COP_Release_2.pdf. [Accessed 13 February 2006].

17. Ibid, 1.

18. COUNTER, Code of Practice for Journals and Databases, Release 2, Appendix E, p. 1-8.

19. COUNTER, Code of Practice for Journals and Databases, Release 2, Introduction. April 2005. Available online at http://www.projectcounter.org/r2/Introduction_to_Release_2_for_Journals_ and_Databases.pdf. [Accessed 13 February 2006].

20. Peter Shepherd, "Impact of the COUNTER Code of Practice on Vendor Usage Statistics", e-mail posting to LIBLICENSE-L, 18 June 2004. Available online at http://www.library.yale. edu/ llicense/ListArchives/0406/msg00112.html. [Accessed 6 February 2006].

21. COUNTER, Code of Practice for Journals and Databases, Release 2, Appendix A: Glossary of Terms, 3.1.4.3, "Timeout."

22. COUNTER, Code of Practice for Journals and Databases, Release 2, Appendix E, p. 5. 
23. Philip M. Davis and Jason S. Price, “EJournal Interface Can Influence Usage Statistics: Implications for Libraries, Publishers, and Project COUNTER," JASIST 57 (July 2006): 1243-48.

24. National Information Standards Organization, "NISO Standardized Usage Statistics Harvesting Initiative (SUSHI)." Available online at http://www.niso.org/committees/SUSHI/SUSHI_comm.html. [Accessed 10 May 2006].

25. MPS Technologies, "ScholarlyStats Overview." Available online at https://www.scholarlystats.com/sstats/default.htm. [Accessed 10 May 2006].

26. Doug Newman, “Journal Use Reports-Making Collection Development Easier," KnowledgeLink Newsletter, January/February 2006. Available online at http://thomsonscientific.com/ news/newsletter/2006-01/8310175/. [Accessed 2 May 2006].

27. Deborah D. Blecic, Joan B. Fiscella, and Stephen E. Wiberley, Jr., "The Measurement of Use of Web-based Information Resources: An Early Look at Vendor-Supplied Data," College E Research Libraries 62 (Sept. 2001): 434-53.

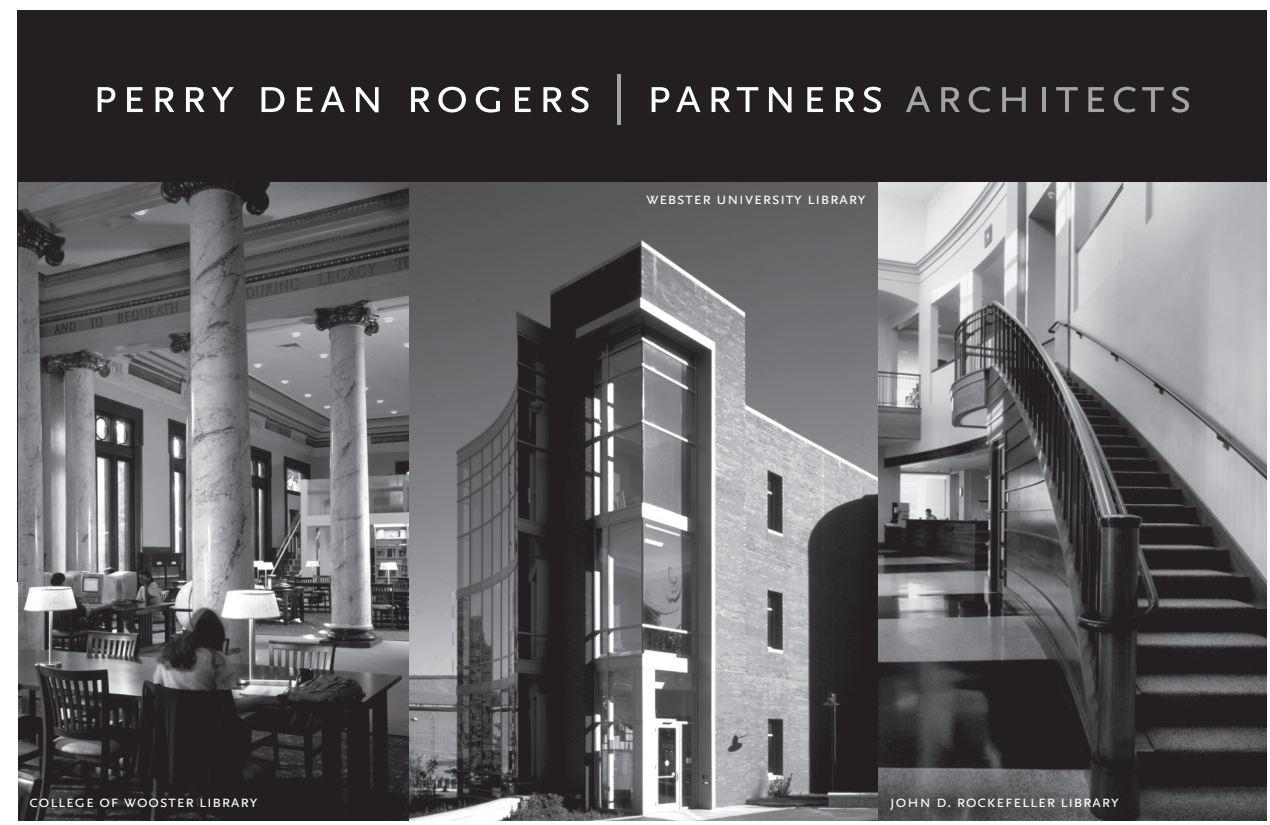

Designers for Libraries \& Academic Institutions 